\title{
1,2,4-三唑取代的苯甲酰胺衍生物的合成及其抑菌活性
}

\author{
蒋振华 $a$ 程绎南 $*, a, b$ 申国富 ${ }^{a}$ 张蒙萌 ${ }^{a}$ \\ 苏子洋 ${ }^{a}$ 孙连省 ${ }^{a}$ 李洪连 $a, b$ \\ ( ${ }^{a}$ 河南农业大学植保学院 郑州 450002) \\ $\left({ }^{b}\right.$ 新型农药创制与应用河南省重点实验室 郑州 450002)
}

\begin{abstract}
摘要 设计了 1,2,4-三唑苯甲酰胺衍生物的合成路线，该路线包括 2,6-二氯苯腈与 1,2,4-三唑的催化偶联、腈的酰胺化、 酰胺重氮化水解及酸的酰胺化等步骤. 采用该路线合成了 14 个未见文献报道的 1,2,4-三唑苯甲酰胺衍生物, 其结构用 ${ }^{1} \mathrm{H}$ NMR、 ${ }^{13} \mathrm{C}$ NMR 和 HRMS 进行了表征; 利用平皿法测试了它们对小麦全蚀病和小麦根腐病病原菌的抑制活性. 结 果表明, 化合物 2-氯- $N$-苯基-6-( $1 H-1,2,4$-三唑- 1 基)苯甲酰胺(7i)在 $100 \mathrm{mg} / \mathrm{L}$ 浓度下对供试小麦全蚀病病原菌抑制率达 到 $80 \%$, 在 50 和 $25 \mathrm{mg} / \mathrm{L}$ 的浓度下抑制率接近对照硅噻菌胺的水平, 而对能引起小麦根腐病的假禾谷镰刀菌的抑制活 性不显著.
\end{abstract}

关键词 1,2,4-三唑苯甲酰胺衍生物; 合成; 小麦全蚀病病原菌; 抑菌活性

\section{Synthesis of 1,2,4-Triazole Benzamide Derivatives and Fungicidal Activity}

\author{
Jiang, Zhenhua ${ }^{a} \quad$ Cheng, Yi'nan $^{*, a, b} \quad$ Shen, Guofu ${ }^{a} \quad$ Zhang, Mengmeng ${ }^{a}$

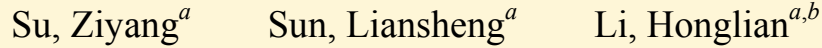 \\ ( ${ }^{a}$ Plant Protection College, Henan Agricultural University, Zhengzhou 450002) \\ ( ${ }^{b}$ Provincial Key Laboratory of the Discovery and Application of Novel Pesticide, Zhengzhou 450002)
}

\begin{abstract}
The synthetic route of substituted 1,2,4-triazole benzamide derivatives was explored, which included several steps such as catalytic cross-coupling of 2,6-dichlorobenzonitrile with a triazole, amidation of nitrile, diazotization of amide and hydrolysis, and amidation of acid. Unreported 14 new 1,2,4-triazole benzamide derivatives were synthesized. Their chemical structures were characterized by ${ }^{1} \mathrm{H}$ NMR, ${ }^{13} \mathrm{C}$ NMR and HRMS. Their antifungal activities against Gaeumannomyces graminis var. tritici and Fusarium pseudocerealum were evaluated in vitro by the plate method. The results indicated that antifungal activities of compound 2-chloro- $N$-phenyl-6-(1H-1,2,4-triazol-1-yl)benzamide (7i) against Gaeumannomyces graminis var. tritici reached up to $80 \%$ at the concentrations of $100 \mathrm{mg} / \mathrm{L}$, and were comparable to the control level of silthiopham at the concentrations of 50 and $25 \mathrm{mg} / \mathrm{L}$. However, these compounds didn't show obvious antifungal activities against Fusarium pseudocerealum.
\end{abstract}

Keywords 1,2,4-triazobenzamide derivatives; synthesis; Gaeumannomyces graminis var. tritici; antifungal activity

芳甲酰胺类衍生物是具有广泛生物活性的一类化 合物，多年来备受关注. 苯甲酰衍生物在医药领域发挥 着重要的作用, 取代的苯甲酰胺在精神分裂症抑制 ${ }^{[1]}$ 、 癌细胞的控制 ${ }^{[2-3]}$ 、对感染 HIV-1 潜伏期细胞的清除 ${ }^{[4]}$ 等方面发挥良好活性. 一些结构的苯甲酰胺作为鱼尼丁 受体(RyR)抑制剂具有优良的杀虫活性 ${ }^{[5-8]}$, 已经商品化
的有氯虫苯甲酰胺(Chlorantraniliprole $)^{[9]}$ 、溴氰虫酰胺 (Cyantraniliprole) $)^{[10]}$ 等(图 1). 在除草剂领域, 苯甲酰胺 衍生物可以抑制乙酰乳酸合成酶(AHAS)，从而发挥除 草活性 ${ }^{[1]}$. 近年来, 芳甲酰胺类衍生物在杀菌剂领域的 研究也比较活跃，一系列的芳甲酰胺化合物被开发为杀 菌剂 ${ }^{[12]}$, 如商品化的品种有啶酰菌胺(Boscalid) ${ }^{[13]}$ 、氟啶

\footnotetext{
* Corresponding author. E-mail: chyn212@aliyun.com

Received March 16, 2020; revised April 17, 2020; published online May 19, 2020.

Project supported by the National Natural Science Foundation of China (No. U1704116) and the National Key R\&D Program of China (No. 2017YFD0201700).

国家自然科学基金(No. U1704116)和国家重点研发计划(No. 2017YFD0201700)资助项目.
} 
酰菌胺(Fluopicolide) $)^{[14]}$ 、吡噻菌胺(Penthiopyrad $)^{[15]}$ 、硅 噻菌胺(Silthiopham) ${ }^{[16]}$ 等(图 1).<smiles>CNC(=O)c1cc(Cl)cc(C)c1NC(=O)c1cc(Br)nn1-c1ncccc1Cl</smiles>

Chlorantraniliprole<smiles>O=C(Nc1ccccc1-c1ccc(Cl)cc1)c1cccnc1Cl</smiles>

Boscalid<smiles>C=CCNC(=O)c1c([Si](C)(C)C)sc(C)c1C</smiles>

Silthiopham<smiles>CNC(=O)c1cc(C#N)cc(C)c1NC(=O)c1cc(Br)nn1-c1ncccc1Cl</smiles>

Cyantraniliprole<smiles>CC(C)CC(C)c1sccc1NC(=O)c1cn(C)nc1C(F)(F)F</smiles><smiles>O=C(NCc1ncc(C(F)(F)F)cc1Cl)c1c(Cl)cccc1Cl</smiles>

Fluopicolide
图 1 芳甲酰胺类活性化合物的结构

Figure 1 Structures of aryl formamide active compounds

小麦全蚀病是危害小麦的一种顽固土传病害 ${ }^{[17]}$, 大多数杀菌剂对其防治效果不够理想, 在目前的杀菌剂 品种中, 硅塞菌胺是少数能够有效防治小麦全蚀病的芳 甲酰胺类杀菌剂, 但品种单一. 为了解决小麦全蚀病防 治品种单一的问题, 并结合芳甲酰胺衍生物在不同生物 学领域良好的生物活性表现, 课题组近来也开展了芳甲 酰胺类化合物的合成与活性研究 ${ }^{[18-19]}$, 发现具有不同结 构的噻吩甲酰胺衍生物对小麦全蚀病具有优异的抑制 活性, 然而有关取代的苯甲酰胺衍生物对防治小麦全蚀 病的活性研究还涉及得较少; 作为该工作的进一步延 伸, 本文拟设计合成一系列取代的苯甲酰胺衍生物, 研 究它们的结构与活性关系, 以期获得对小麦全蚀病具有 抑制活性的新先导化合物.

另一方面，1,2,4-三唑官能团在三唑类杀菌剂中扮

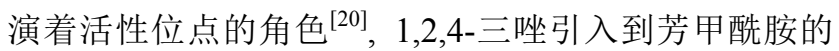
结构中可能衍生出一类新的杀菌活性化合物, 为此, 设 计了具有较新颖结构的 1,2,4-三唑苯甲酰胺衍生物, 如 图 2 所示.

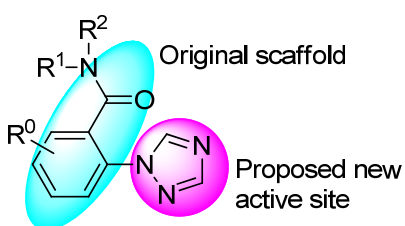

图 2 目标化合物结构

Figure 2 Structure of target compound
为了获得这些化合物，本工作组设计了如 Scheme 1 所示的合成路线，并通过该路线顺利地合成了 14 个未 见文献报道的 1,2,4-三唑苯甲酰胺衍生物, 分别考察了 它们对小麦全蚀病病原菌(Gaeumannomyces graminis var. tritici)和小麦根腐病病原菌(Fusarium pseudocerealum) 的抑制活性，初步分析了所合成化合物的结构活性 关系.
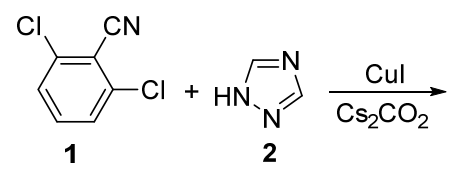<smiles>N#Cc1c(Cl)cccc1-n1cncn1</smiles>
$\mathrm{H}_{2} \mathrm{SO}_{4}$<smiles>NC(=O)c1c(Cl)cccc1-n1cncn1</smiles><smiles>O=C(O)c1c(Cl)cccc1-n1cncn1</smiles>

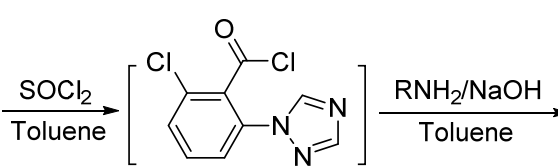

6

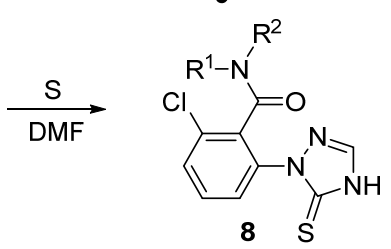

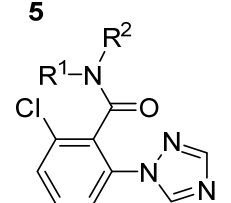

$7 a \sim 71$
$4 \mathrm{R}^{1}=\mathrm{H}, \mathrm{R}^{2}=\mathrm{H} ; \mathbf{7 a} \mathrm{R}^{1}=\mathrm{H}, \mathrm{R}^{2}=$ allyl; $7 \mathrm{~b} \mathrm{R}^{1}=\mathrm{H}, \mathrm{R}^{2}=$ isopropyl; 7c $\mathrm{R}^{1}=$ $\mathrm{H}, \mathrm{R}^{2}=$ cyclopropylmethyl; 7d $\mathrm{R}^{1}=\mathrm{H}, \mathrm{R}^{2}=$ 2-chlorbenzyl; 7e $\mathrm{R}^{1}=\mathrm{H}$, $\mathrm{R}^{2}=4$-chlorobenzyl; 7f $\mathrm{R}^{1}=\mathrm{H}, \mathrm{R}^{2}=4$-methoxybenzyl; $7 \mathrm{~g} \mathrm{R}^{1}=\mathrm{Et}, \mathrm{R}^{2}=$ Et; $7 h R^{1}=H, R^{2}=E t ; 7 i \quad R^{1}=H, R^{2}=$ benzene; $7 j R^{1}=H, R^{2}=$ 2-chlorobenzene; $\mathbf{7 k} \mathrm{R}^{1}=\mathrm{H}, \mathrm{R}^{2}=$ 2-fluorobenzene; $7 \mathrm{I}^{1}=\mathrm{H}, \mathrm{R}^{2}=$ 2-cyanobenzene; $8 \mathrm{R}^{1}=\mathrm{H}, \mathrm{R}^{2}=2$-chlorobenzyl

图式 1 目标化合物的合成路线

Scheme 1 Synthetic routes of target compounds

\section{1 结果与讨论}

\subsection{2-氯-6-(1,2,4-三唑-1-基)苯甲腈(3)的合成}

在目标化合物的合成过程中，苯环上三唑官能团的 引入具有一定的挑战, 同时涉及到原料的选取. 由于苯 环 1,2,4-三唑的引入通常可以通过碳-氮键的催化偶联 来实现 ${ }^{[21]}$, 因此目标化合物的逆合成分析如 Scheme 2 所示.

首先尝试以容易获得的 2,6-二氯苯甲酸为原料，考 察通过催化偶联引入 1,2,4-三唑的可能性, 然而在选定 的条件下 ${ }^{21]}$ ，几乎没有目标产物生成. 考虑到䧳基对反 应的可能影响，考察了以 2,6-二氯苯甲酸酯为原料来实 现偶联的可能性，尝试了醋酸钯、三苯基膦钯 ${ }^{[22]}$ 、碘化 亚铜、溴化亚铜 ${ }^{[21]}$ 等与不同的碱组合的催化体系，也没 有获得理想的催化偶联效果，却得到了脱羰基化产物. 


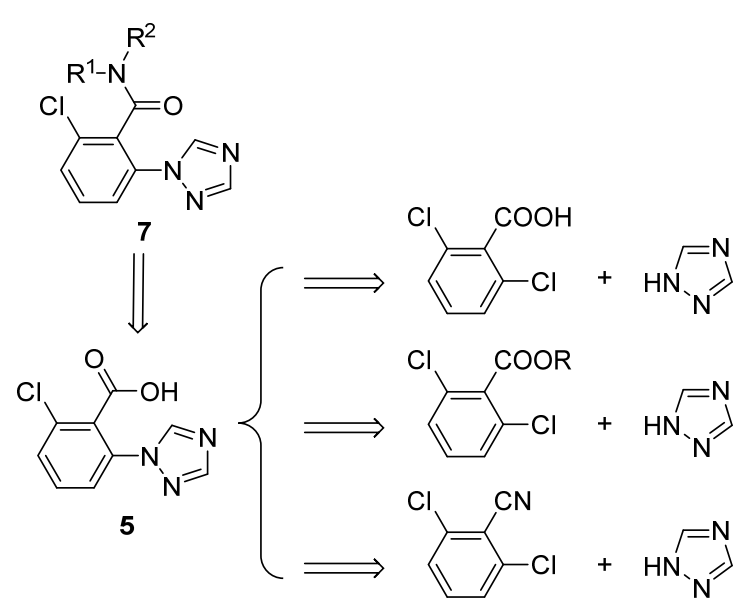

图式 2 目标化合物的逆合成分析

Scheme 2 Retrosynthetic analysis of target compounds

可能是 2,6-二氯苯甲酸酯的碳-氯键与 $1,2,4-$ 三唑的偶联 活性低 ${ }^{[23]}$, 酯基又不能承受选定的反应条件等. 拟通过 改变底物提高其反应活性以促进反应的进行, 选择了以 2,6-二氯苯腈为原料的合成路线, 取得了进展. 针对该 合成方法进行了催化剂选择和反应条件优化, 结果如表 1 所示.

表 1 2-氯-6-(1,2,4-三唑-1-基)苯甲腈的合成与条件优化 ${ }^{a}$ Table 1 Synthesis of 2-chloro-6-(1H-1,2,4-triazol-1-yl)benzonitrile and conditions optimizing

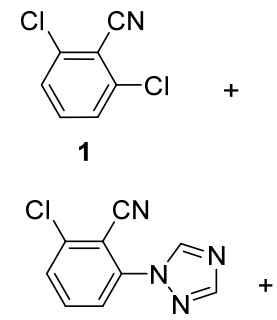

3
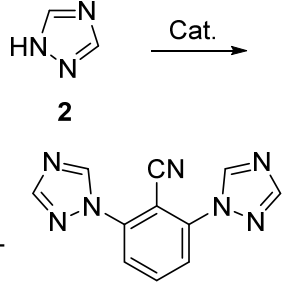

$3^{\prime}$

\begin{tabular}{ccccccc}
\hline Entry & \multicolumn{2}{c}{ Promoter Catalysis } & $T /^{\circ} \mathrm{C}$ & Time $/ \mathrm{h}$ & $\mathbf{3}: \mathbf{3}^{b}$ & Yield $/ \%^{c}$ \\
\hline 1 & $\mathrm{~K}_{2} \mathrm{CO}_{3}$ & - & 110 & 20 & $1: 1$ & 40 \\
2 & $\mathrm{Cs}_{2} \mathrm{CO}_{3}$ & - & 110 & 20 & $1: 1.6$ & 30 \\
3 & $\mathrm{Cs}_{2} \mathrm{CO}_{3}$ & $\mathrm{CuI} / \mathrm{M}^{d}$ & 110 & 12 & $3.4: 1$ & 63 \\
4 & $\mathrm{Cs}_{2} \mathrm{CO}_{3}$ & $\mathrm{CuI} / \mathrm{M}^{d}$ & 85 & 12 & $3.5: 1$ & 65 \\
5 & $\mathrm{Cs}_{2} \mathrm{CO}_{3}$ & $\mathrm{CuI} / \mathrm{M}^{d}$ & 65 & 12 & $3.2: 1$ & 52 \\
6 & $\mathrm{Cs}_{2} \mathrm{CO}_{3}$ & $\mathrm{CuBr} / \mathrm{M}^{d}$ & 85 & 12 & $3: 1$ & 60 \\
\hline
\end{tabular}

${ }^{a} 1$ equiv. of 2,6-dichlorobenzonitrile, 1.3 equiv. of 1,2,4-triazole and alkali carbonates were used in $3 \mathrm{~mL}$ of $N, N$-dimethylformamide (DMF). ${ }^{b}$ The molar ratio of $3: 3^{\prime}$ was measured by HPLC. ${ }^{c}$ Isolated yields of $\mathbf{3}$ based on 2, 6-dichlorobenzonitrile. ${ }^{d} 0.05$ equiv. of copper salt and M (8-hydroxyquinoline- $N$-oxide).

2,6-二氯苯甲腈与 1,2,4-三唑偶联表现出了相对较 高的反应活性, 在以 $\mathrm{N}, \mathrm{N}$-二甲基甲酰胺为溶剂, 碳酸钾 为碱, 在 $110{ }^{\circ} \mathrm{C}$ 的条件下反应 $20 \mathrm{~h}$, 原料大部分发生转 化, 遗憾的是反应的选择性较差, 1,2,4-三唑单取代物 3 和二取代产物 3 '同时生成, 生成量几乎相等, 目标产物 只得到 $40 \%$ 的收率(Entry 1). 以碳酸铯为碱时, 1,2,4-三
唑二取代产物 $3^{\prime}$ 占优(Entry 2). 为了提高反应的选择性, 尝试了在铜盐及促进剂存在下的碳-氮偶联反应 ${ }^{[24]}$. 结 果发现, 以 $\mathrm{Cs}_{2} \mathrm{CO}_{3}$ 为碱、 $\mathrm{CuI} / 8$-差基喹啉氮氧化物为催 化剂的反应体系, 在 $110{ }^{\circ} \mathrm{C}$ 反应 $12 \mathrm{~h}$, 原料基本完全转 化，反应的选择性具有明显改善，1,2,4-三唑单取代物 3 和二取代产物 3'的比率提高到 $3.4: 1$, 目标产物的收率 达到中等水平 $(63 \%$, Entry 3$)$. 为了进一步提高选择性, 把温度降低到 $85{ }^{\circ} \mathrm{C}$, 反应亦可顺利进行, 收率稍有改 善, 达到 $65 \%$ 的水平(Entry 4). 进一步将反应温度降至 $65{ }^{\circ} \mathrm{C}$, 反应转化不完全. 变换催化剂的种类为溴化亚 铜, 反应收率及选择性在所选条件下没有进一步改善 (Entry 6). 较高的 1,2,4-三唑和碱的用量, 会降低反应的 选择性，而较低的 1,2,4-三唑和碱的用量导致 2,6-二氯 苯腈转化率降低. 归纳比较优惠的反应条件为: 以 $N, N$ 二甲基甲酰胺(DMF)为溶剂, 1.3 equiv. $\mathrm{Cs}_{2} \mathrm{CO}_{3}$ 为碱, 0.05 equiv. $\mathrm{CuI} / 8$-着基喹啉氮氧化物为催化剂, 在 $85{ }^{\circ} \mathrm{C}$ 反应 $12 \mathrm{~h}$.

\subsection{2-氯-6-(1,2,4-三唑-1-基)苯甲酸的合成}

首先尝试在碱性条件下直接将氰基水解为酸的方 法 $^{[25]}$, 然而, 氢氧化钠、氢氧化钾和甲醇钠等在回流条 件下都未能较好地实现氧基向羧酸的转化. 转而考察在 硫酸存在下将氰基水解为酸的方法 ${ }^{[26]}$, 遗憾的是反应 仅停留在中间体 4 的步骤. 有文献报道, 酰胺可以在 $\mathrm{NaNO}_{2} / \mathrm{H}_{2} \mathrm{SO}_{4}$ 的存在下平稳地水解为羧酸 ${ }^{[27]}$, 于是采取 两步法来实现氰基向羧酸的转化, 取得了进展. 首先中 间体 3 在浓度为 $80 \%$ 的硫酸水溶液中在 $100{ }^{\circ} \mathrm{C}$ 反应 10 $\mathrm{h}$, 实现了氧基向酰胺的完全转化得到中间体 4. 然后, 在低温下 $\left(0{ }^{\circ} \mathrm{C}\right.$ 左右 $)$ 向含有中间体 $\mathbf{4}$ 的 $80 \%$ 硫酸水溶液 中加入 5 equiv.亚硝酸钠水溶液后反应 $1 \mathrm{~h}$, 使酰胺氮原 子发生重氮化，接着再在 $100{ }^{\circ} \mathrm{C}$ 反应 $2 \mathrm{~h}$, 以 $84 \%$ 的收 率得到中间体 2-氯-6-(1,2,4-三唑-1-基)苯甲酸(5).

\subsection{2 -氯-6-(1,2,4-三唑-1-基)苯甲酰胺(7a～7I)和化合 物 8 的合成}

目标化合物 7a 71 的合成参照文献[19]进行. 首先, 合成的 2-氯-6-(1,2,4-三唑-1-基)苯甲酸(5)在氯化亚砜的 存在下, 转化为相应的 2-氯-6-(1,2,4-三唑-1-基)苯甲酰 氯(6), 苯甲酰氯 6 不需分离直接在缚酸剂氢氧化钠的存 在下, 分别与各种取代的胺缩合得到 2-氯-6-(1,2,4-三 唑-1-基)苯甲酰胺衍生物 7a 7 . 由于苯甲酰氯 6 在各 种取代胺的存在下, 对 $10 \%$ 氢氧化钠水溶液不敏感，使 用低浓度的氢氧化钠水溶液作为缚酸剂, 反应的后处理 更加容易. 化合物 8 则由化合物 7d 在硫磺的存在下在 $N, N$-二甲基甲酰胺中通过加热硫化而得 ${ }^{[28]}$.

目标化合物的表征主要关注 1,2,4-三唑官能团在结 构中是否存在，由于 $1,2,4$-三唑官能团的两个质子和两 
个碳原子分别在 ${ }^{1} \mathrm{H}$ NMR 和 ${ }^{13} \mathrm{C}$ NMR 中以比较特征的 信号在低场出现, 因此, 在目标化合物中 1,2,4-三唑官 能团都清晰可辨, 其它质子和碳原子信号(除 $7 \mathbf{k}$ 外)也都 与结构完全一致. 至于化合物 $7 \mathbf{k}$ 的结构表征, 高分辨质 谱 $[\mathrm{M}+\mathrm{H}]^{+}$对应的质荷比为 317.0603 , 与理论值 317.0605 符合得很好. 其 ${ }^{1} \mathrm{H}$ NMR 化学位移在 $\delta 10.51$ 处的质子信号对应酰胺氮原子的质子, 而 $\delta 8.90$ 和 8.21 处的质子信号分别对应三唑官能团的两个质子; 化学位 移 $\delta$ 在 7.73 7.69 和 7.26 7.19 处的两组质子信号对应 两个苯环的 7 个质子, 质子信号与结构也符合得较好. 由于氟原子的存在, 化合物 7k 的 ${ }^{13} \mathrm{C} \mathrm{NMR}$ 谱中存在着 F-C 偶合现象, 氟原子与含氟苯环的 6 个碳原子分别发 生偶合, 致使含氟苯环的每个碳原子都显示两个信号, 并呈现出不同的偶合常数, 如 $\delta 156.0$ 和 153.5 处的碳信 号, 对应与氟直接相连的碳原子, 偶合常数为 $245.5 \mathrm{~Hz}$; $\delta 126.9$ 和 126.8 处的碳信号, 对应氟原子邻位与氮原子 相连的碳原子, 偶合常数为 $7.4 \mathrm{~Hz}$. 谱图呈现的这种偶 合裂分现象表明氟原子的存在, 也体现了谱图与结构的 一致性.

\section{4 三唑苯甲酰胺衍生物结构与活性关系初步分析}

为了评估三坐苯甲酰胺衍生物的结构活性关系, 采 用平皿菌丝生长速率法 ${ }^{[29]}$ 测定了所合成新化合物 $\mathbf{4}$ 和 7a 71 对小麦全蚀病病原菌(Gaeumannomyces graminis var. tritici) 的抑制活性, 测试结果如表 2 所示. 起初, 噻 吩甲酰胺氮原子上的取代基为烯丙基和烷基时, 表现出 较高活性的结果 ${ }^{[18]}$, 将烯丙基和具有代表性的含有 2 4 个碳的简单烷基引入到三唑苯甲酰胺的酰胺氮原子
上, 但其活性表现较低 $(\mathbf{7 a} \sim 7 \mathbf{c}, 7 \mathbf{g} \sim 7 \mathbf{h})$, 在 $100 \mathrm{mg} / \mathrm{L}$ 的浓度下，其抑制率最高仅为 $30 \%$ 左右. 当酰胺氮原子 上的取代基变成取代的苯甲基时(7d 7f $)$, 其活性总体 上具有增加的趋势. 其中 $7 \mathbf{f}$ 在 $100 \mathrm{mg} / \mathrm{L}$ 的浓度下，对小 麦全蚀病病原菌的抑制率超过了 $50 \%$. 这进一步支持优 化酰胺氮原子上的取代基，当酰胺氮原子上的取代基变 为苯基时, 其活性有明显提高, 化合物 $7 \mathbf{i}$ 在 $100 \mathrm{mg} / \mathrm{L}$ 的 浓度下, 对小麦全蚀病的供试病原菌抑制率达到了 $80 \%$; 在 50 和 $25 \mathrm{mg} / \mathrm{L}$ 的浓度下, 其抑制率分别为 $63.7 \%$ 和 $43.6 \%$, 接近对照硅噻菌胺的水平. 于是利用现 有的取代苯胺又合成了 $7 \mathbf{j} \sim 7 \mathbf{7}$, 活性测试结果表明, 它 们也表现出了相对较高的活性, 在 $100 \mathrm{mg} / \mathrm{L}$ 的浓度下 分别达到 61.7\%, 69.7\%和 65.4\%的抑制率. 作为对比, 酰胺氮原子上无取代基时，如化合物 $\mathbf{4}$, 其活性不及取 代基为苯的活性. 总体分析, 所合成三唑苯甲酰胺衍生 物对小麦全蚀病病原菌均具有一定的抑制活性，当酰胺 氮原子上无取代基或为烷基单取代、双取代时，均不利 于活性的产生, 酰胺氮原子上的取代基为各种取代的苯 甲基时, 活性增加, 尤以取代的苯基时活性更好，值得 进一步的优化研究.

为了进一步了解 1,2,4-三唑苯甲酰胺衍生物的活性 范围, 本工作还测试了它们对小麦根腐病病原菌假禾谷 镰狍菌(Fusarium pseudocerealum)的抑制活性, 令人遗 憾的是, 所合成的 $1,2,4$-三唑苯甲酰胺衍生物, 酰胺氮 原子上的取代基无论是烷基还是芳基，在设计浓度 $(100,50,25 \mathrm{mg} / \mathrm{L})$ 下虽然都表现出了一定的活性, 但活 性都较低, 只有 71 表现出了相对较好的活性, 在 100

表 2 1,2,4-三唑苯甲酰胺衍生物的抑菌活性测试(抑制率 $/ \%)^{a}$

Table 2 Inhibitory activity (Inhibition rate/\%) of 1,2,4-triazole benzamide derivatives

\begin{tabular}{|c|c|c|c|c|c|c|}
\hline \multirow{2}{*}{ Compd. } & \multicolumn{3}{|c|}{ Gaeumannomyces graminis var. tritici } & \multicolumn{3}{|c|}{ Fusarium pseudocerealum } \\
\hline & $100 \mathrm{mg} / \mathrm{L}$ & $50 \mathrm{mg} / \mathrm{L}$ & $25 \mathrm{mg} / \mathrm{L}$ & $100 \mathrm{mg} / \mathrm{L}$ & $50 \mathrm{mg} / \mathrm{L}$ & $25 \mathrm{mg} / \mathrm{L}$ \\
\hline 4 & 46.0 & 20.2 & 16.0 & 10.8 & 7.7 & 4.6 \\
\hline $7 \mathbf{a}$ & 19.6 & 14.2 & 5.8 & 4.8 & 3.9 & 1.7 \\
\hline $7 b$ & 12.5 & 9.6 & 7.5 & 12.1 & 6.1 & 5.6 \\
\hline $7 \mathrm{c}$ & 10.4 & 5.8 & 3.8 & 27.5 & 16.7 & 6.9 \\
\hline 7d & 44.7 & 21.1 & 13.2 & 6.2 & 4.4 & 3.4 \\
\hline $7 e$ & 36.4 & 34.2 & 12.4 & 10.4 & 7.7 & 5.0 \\
\hline $7 f$ & 50.9 & 21.9 & 12.3 & 21.9 & 16.4 & 10.1 \\
\hline $7 \mathrm{~g}$ & 30.3 & 25.4 & 17.5 & 33.8 & 30.9 & 29.4 \\
\hline $7 \mathrm{~h}$ & 32.1 & 17.1 & 7.3 & 20.6 & 19.9 & 17.5 \\
\hline $7 \mathbf{i}$ & 80.0 & 63.7 & 43.6 & 12.8 & 9.4 & 7.3 \\
\hline $7 \mathbf{j}$ & 61.7 & 34.7 & 15.3 & 39.6 & 30.2 & 17.6 \\
\hline $7 k$ & 69.7 & 53.3 & 32.0 & 31.6 & 24.4 & 16.0 \\
\hline 71 & 65.4 & 50.4 & 25.8 & 44.6 & 34.2 & 22.9 \\
\hline 8 & - & - & - & 28.1 & 24.6 & 21.9 \\
\hline Silthiopham & 92.3 & 66.7 & 40.5 & - & - & - \\
\hline Prothioconazole & 100 & 97.3 & 94.1 & 100 & 100 & 100 \\
\hline
\end{tabular}

a "-_"No detection. 
$\mathrm{mg} / \mathrm{L}$ 的浓度下给出大约 $45 \%$ 的抑制率. 由于三唑硫酮 衍生物 ${ }^{[30]}$ 对假禾谷镰狍菌 (Fusarium pseudocerealum) 具 有优异的抑制活性, 本工作又合成了三唑硫酮取代的苯 甲酰胺衍生物 $\mathbf{8}$, 但该化合物没有表现出期待的活性. 根据目前三唑苯甲酰胺衍生物结构与活性的表现, 进一 步的结构优化是值得尝试的, 相关工作在进行中.

\section{2 结论}

设计了以 2,6-二氯苯腈和 1,2,4-三唑为原料合成 1,2,4-三唑苯甲酰胺衍生物的工艺方法, 优化了该方法 中 2,6-二氯苯腈和 1,2,4-三唑催化偶联的反应条件, 提 高了反应的选择性. 开发了中间体 1,2,4-三唑取代的苯 腈两步法水解为羧酸的方法, 顺利地获得了中间体 $1,2,4$-三唑取代的苯甲酸. 通过 1,2,4-三唑取代的苯甲酸 的酰化与胺化, 合成了 14 个三唑苯甲酰胺衍生物. 活性 测试表明, 除化合物 8 外, 大部分化合物对供试小麦全 蚀病病原菌都表现出一定的抑制活性, 尤以化合物 $7 \mathbf{i}$ 表 现最好, 在 $100 \mathrm{mg} / \mathrm{L}$ 的浓度下, 抑制率达到了 $80 \%$. 在 50 和 $25 \mathrm{mg} / \mathrm{L}$ 的浓度下, 抑制率接近对照硅噻菌胺的水 平. 初步结构活性关系分析表明, 所合成 1,2,4-三唑苯 甲酰胺衍生物酰胺氮原子芳基取代的活性优于酰胺氮 原子烷基取代的活性, 这为活性化合物结构的进一步设 计提供了一定的参考.

\section{3 实验部分}

\section{1 仪器与试剂}

反应使用 HPLC (Agilent-1400) 检测和分析; ${ }^{1} \mathrm{H}$ NMR、 ${ }^{13} \mathrm{C}$ NMR 用核磁共振仪 (Agilent-NMR-vnmrs 400) 测试, 以 $\mathrm{CDCl}_{3}$ 或 DMSO- $d_{6}$ 为溶剂, TMS 为内标; 质谱 用 Bruker micrOTOF-QII 质谱仪测试; 熔点用 WRS-1A 数字熔点仪测试; 病原菌使用 $\mathrm{HPG}-280 \mathrm{H}$ 人工气候箱培 养. 所有国产或进口 AR 或 CP 级试剂未经纯化直接使 用.

\section{2 病原菌菌株}

供试的小麦全蚀病病原菌(Gaeumannomyces graminis var. tritici) 由南京农业大学植物保护学院实验室提 供; 小麦根腐病病原菌假禾谷镰狍菌(Fusarium pseudocerealum) 由河南农业大学植物保护学院病理实验室提 供.

\section{3 中间体及目标化合物的合成}

3.3.1 2-氯-6-(1H-1,2,4-三唑-1-基)苯甲腈(3)的合成

将 $0.17 \mathrm{~g}(1 \mathrm{mmol})$ 2,6-二氯苯甲腈, $0.09 \mathrm{~g}(1.3$ $\mathrm{mmol})$ 工业级 1,2,4-三唑, $0.43 \mathrm{~g}(1.3 \mathrm{mmol})$ 无水碳酸铯 和 $9.5 \mathrm{mg}(0.05 \mathrm{mmol}) \mathrm{CuI}$ 及 $8.1 \mathrm{mg}(0.05 \mathrm{mmol}) 8$-羟基
喹啉- $N$-氧化物配体加入到 $20 \mathrm{~mL}$ 的小反应瓶中, 然后 量取 $3 \mathrm{~mL}$ 的 $N, N$-二甲基甲酰胺加入到反应瓶中, 在油 浴中加热到 $85{ }^{\circ} \mathrm{C}$ 摚拌反应 $12 \mathrm{~h}$. 薄层色谱(TLC)检测反 应完成后，负压滤除碳酸钾以及未反应的 1,2,4-三唑, 滤液水洗萃取后浓缩，用薄层色谱(氯仿/乙醇, $V: V=$ $100 ：$ 1)分离得到 2-氯-6-(1H-1,2,4-三唑-1-基)苯甲腈(3) $133 \mathrm{mg}$, 收率 79\%. 亚白色固体, m.p. $170 \sim 172{ }^{\circ} \mathrm{C} ;{ }^{1} \mathrm{H}$ NMR $\left(400 \mathrm{MHz}, \mathrm{CDCl}_{3}\right) \delta: 8.79(\mathrm{~s}, 1 \mathrm{H}), 8.20(\mathrm{~s}, 1 \mathrm{H})$, $7.74 \sim 7.69(\mathrm{~m}, 2 \mathrm{H}), 7.64 \sim 7.62(\mathrm{~m}, 1 \mathrm{H}) ;{ }^{13} \mathrm{C}$ NMR $(100$ $\left.\mathrm{MHz}, \mathrm{CDCl}_{3}\right) \delta: 153.3,143.3,140.3,139.2,134.5,129.8$, 123.1, 113.3, 107.7. HRMS (ESI) calcd for $\mathrm{C}_{9} \mathrm{H}_{6} \mathrm{ClN}_{4}$ $[\mathrm{M}+\mathrm{H}]^{+}$205.0281, found 205.0275.

2,6-二(1H-1,2,4-三唑-1-基)苯甲腈 $\left(3^{\prime}\right)$ : 亚白色固体, m.p. $245 \sim 246{ }^{\circ} \mathrm{C} ;{ }^{1} \mathrm{H}$ NMR $\left(400 \mathrm{MHz}, \mathrm{CDCl}_{3}\right) \delta: 8.81$ (s, $2 \mathrm{H}), 8.24(\mathrm{~s}, 2 \mathrm{H}), 7.97 \sim 7.93(\mathrm{~m}, 1 \mathrm{H}), 7.87 \sim 7.85(\mathrm{~m}$, $2 \mathrm{H}) ;{ }^{13} \mathrm{C}$ NMR $\left(100 \mathrm{MHz}, \mathrm{CDCl}_{3}\right) \delta: 153.5,143.5,140.5$, 135.2, 125.1, 113.1. HRMS (ESI) calcd for $\mathrm{C}_{11} \mathrm{H}_{8} \mathrm{~N}_{7}[\mathrm{M}+$ $\mathrm{H}]^{+}$238.0841, found 238.0833.

3.3.2 2-氯-6-(1H-1,2,4-三唑-1-基)苯甲酰胺(4)的合成

将 2-氯-6-(1H-1,2,4-三唑-1-基)苯甲腈 $6.1 \mathrm{~g}$ (30 $\mathrm{mmol}$ )加入到三口烧瓶中, 然后加入 $30 \mathrm{~g} 80 \%$ 的硫酸 $(0.24 \mathrm{~mol})$ 水溶液, 加热到 $100{ }^{\circ} \mathrm{C}$ 回流反应 $10 \mathrm{~h}$, 反应呈 暗红色均相溶液, TLC 检测反应完全, 加水调节 $\mathrm{pH}$ 至中 性，用乙酸乙酯萃取干燥，负压蒸出溶剂得到粗品为灰 色固体, 经硅胶柱色谱(氯仿/乙醇, $V: V=100: 1)$ 分离 得到纯品 2-氯-6-(1H-1,2,4-三唑-1-基)苯甲酰胺(4) $5.3 \mathrm{~g}$, 收率 79\%. 灰色固体, m.p. $128 \sim 130{ }^{\circ} \mathrm{C} ;{ }^{1} \mathrm{H}$ NMR $(400$ $\left.\mathrm{MHz}, \mathrm{CDCl}_{3}\right) \delta: 8.52(\mathrm{~s}, 1 \mathrm{H}), 8.11(\mathrm{~s}, 1 \mathrm{H}), 7.58 \sim 7.56(\mathrm{~m}$, $1 \mathrm{H}), 7.53 \sim 7.49$ (m, 2H), 5.86 (brs, $2 \mathrm{H}) ;{ }^{13} \mathrm{C}$ NMR $(100$ $\left.\mathrm{MHz}, \mathrm{CDCl}_{3}\right) \delta: 165.7,152.7,144.2,135.2,132.1,131.5$, 131.1, 130.5, 124.0. HRMS (ESI) calcd for $\mathrm{C}_{9} \mathrm{H}_{8} \mathrm{ClN}_{4} \mathrm{O}$ $[\mathrm{M}+\mathrm{H}]^{+}$223.0387, found 223.0383.

\subsubsection{2-氯-6-(1H-1,2,4-三唑-1-基)苯甲酸(5)的合成}

将 $14.3 \mathrm{~g}$ (0.064 mol) 2-氯-6-(1H-1,2,4-三唑-1-基)苯 甲酰胺加入到 $100 \mathrm{~mL}$ 的三口反应瓶中, 将三口瓶置于 冰浴中, 在 $0 \sim 10{ }^{\circ} \mathrm{C}$ 范围内, 慢慢加入 $79 \mathrm{~g} 80 \%$ 的硫酸 (0.64 mol)水溶液, 滴完后开始滴加已降温至 $0 \sim 10{ }^{\circ} \mathrm{C}$ 的 $22.1 \mathrm{~g}(0.32 \mathrm{~mol})$ 亚硝酸钠配制成的水溶液, 使反应 始终维持在 $0 \sim 10{ }^{\circ} \mathrm{C}$ 之间, 滴加完毕后保温反应 $1 \mathrm{~h}$ 后. 然后再在 $100{ }^{\circ} \mathrm{C}$ 下反应 $2 \mathrm{~h}, \mathrm{TLC}$ 检测反应完全为止. 反 应液用乙酸乙酯萃取、干燥、负压蒸出溶剂后得到淡黄 色 2-氯-6-(1H-1,2,4-三唑-1-基)苯甲酸固体 $12 \mathrm{~g}$, 收率 $84 \%$. 黄色固体, m.p. 122 124 ${ }^{\circ} \mathrm{C} ;{ }^{1} \mathrm{H}$ NMR $(400 \mathrm{MHz}$, DMSO- $\left.d_{6}\right) \delta: 9.03(\mathrm{~s}, 1 \mathrm{H}), 8.26(\mathrm{~s}, 1 \mathrm{H}), 7.74 \sim 7.64(\mathrm{~m}$, $3 \mathrm{H}) ;{ }^{13} \mathrm{C}$ NMR (100 MHz, DMSO- $\left.d_{6}\right) \delta: 165.7,152.9$, 
144.9, 135.0, 131.7, 131.2, 130.4, 130.3, 123.6. HRMS (ESI) calcd for $\mathrm{C}_{9} \mathrm{H}_{7} \mathrm{ClN}_{3} \mathrm{O}_{2}[\mathrm{M}+\mathrm{H}]^{+}$224.0227, found 224.0221

3.3.4 2-氯-6-(1H-1,2,4-三唑-1-基)苯甲酰胺化合物 $7 \mathbf{a} \sim 71$ 的合成

将 $1.4 \mathrm{~g}$ (6.3 mmol) 2-氯-6-(1H-1,2,4-三唑-1-基)苯 甲酸投入到盛有 $20 \mathrm{~mL}$ 甲苯的带有回流和气体吸收装 置的三口反应瓶中, 在 $80{ }^{\circ} \mathrm{C}$ 条件下缓慢加入 $1.5 \mathrm{~g}$ (12.6 mmol)的氯化亚砜, 加完后继续反应 $2 \mathrm{~h}$. 负压脱 出溶剂和过量的氯化亚砜后, 向反应瓶中补加 $10 \mathrm{~mL}$ 新 鲜甲苯, 接着在 $45{ }^{\circ} \mathrm{C}$ 向反应瓶中滴加 $0.5 \mathrm{~g}$ (8.7 mmol) 烯丙胺, 然后向反应液中加入 $3 \mathrm{~g}(7.5 \mathrm{mmol}) 10 \%$ 氢氧 化钠水溶液, 再在 $85{ }^{\circ} \mathrm{C}$ 温度下反应 $2 \mathrm{~h}$, 降温静置分层, 有机相用温水洗涤 $(5 \mathrm{~mL} \times 3)$, 干燥后过滤, 滤液负压蒸 出溶剂, 用硅胶柱色谱(氯仿/乙醇, $V: V=100: 1)$ 分离 得到浅黄色固体. 其它酰胺化合物按相似的方法合成与 分离.

$N$-烯丙基-2-氯-6-(1H-1,2,4-三唑-1-基)苯甲酰胺 (7a): 收率 60\%. 黄色固体, m.p. $130 \sim 131{ }^{\circ} \mathrm{C} ;{ }^{1} \mathrm{H}$ NMR $\left(400 \mathrm{MHz}, \mathrm{CDCl}_{3}\right) \delta: 8.44(\mathrm{~s}, 1 \mathrm{H}), 8.02(\mathrm{~s}, 1 \mathrm{H}), 7.48 \sim$ $7.42(\mathrm{~m}, 3 \mathrm{H}), 5.79$ (brs, $1 \mathrm{H}), 5.72 \sim 5.62(\mathrm{~m}, 1 \mathrm{H}), 5.09 \sim$ $5.05(\mathrm{~m}, 2 \mathrm{H}), 3.89 \sim 3.86(\mathrm{t}, J=6.0 \mathrm{~Hz}, 2 \mathrm{H}) ;{ }^{13} \mathrm{C} \mathrm{NMR}$ $\left(100 \mathrm{MHz}, \mathrm{CDCl}_{3}\right) \delta: 162.9,151.6,143.3,134.4,131.8$, 131.4, 131.1, 129.9, 129.4, 123.0, 116.4, 41.3. HRMS (ESI) calcd for $\mathrm{C}_{12} \mathrm{H}_{12} \mathrm{ClN}_{4} \mathrm{O}[\mathrm{M}+\mathrm{H}]^{+}$263.0700, found 263.0696 .

2-氯- $N$-异丙基-6-(1H-1,2,4-三唑-1-基)苯甲酰胺 (7b): 收率 60\%. 白色固体, m.p. 162 164 ${ }^{\circ} \mathrm{C} ;{ }^{1} \mathrm{H}$ NMR $\left(400 \mathrm{MHz}, \mathrm{CDCl}_{3}\right) \delta: 8.53(\mathrm{~s}, 1 \mathrm{H}), 8.09(\mathrm{~s}, 1 \mathrm{H}), 7.55 \sim$ 7.48 (m, 3H), 5.61 (brs, 1H), $4.19 \sim 4.13(\mathrm{~m}, 1 \mathrm{H}), 1.11 \sim$ $1.09(\mathrm{~d}, J=8.0 \mathrm{~Hz}, 6 \mathrm{H}) ;{ }^{13} \mathrm{C} \mathrm{NMR}\left(100 \mathrm{MHz}, \mathrm{CDCl}_{3}\right) \delta$ : $163.1,152.6,144.3,135.3,132.5,132.4,130.8,130.4$, 124.0, 42.3, 22.3. HRMS (ESI) calcd for $\mathrm{C}_{12} \mathrm{H}_{14} \mathrm{ClN}_{4} \mathrm{O}$ $[\mathrm{M}+\mathrm{H}]^{+}$265.0856, found 265.0850.

2-氯- $N$-(环丙基甲基)-6-(1H-1,2,4-三唑-1-基)苯甲酰 胺(7c): 收率 55\%. 浅灰色固体, m.p. $128 \sim 129{ }^{\circ} \mathrm{C} ;{ }^{1} \mathrm{H}$ NMR (400 MHz, $\left.\mathrm{CDCl}_{3}\right) \delta: 8.53(\mathrm{~s}, 1 \mathrm{H}), 8.08(\mathrm{~s}, 1 \mathrm{H})$, $7.56 \sim 7.49$ (m, 3H), 5.93 (brs, $1 \mathrm{H}), 3.19 \sim 3.16(\mathrm{~m}, 2 \mathrm{H})$, $0.88 \sim 0.84(\mathrm{~m}, 1 \mathrm{H}), 0.49 \sim 0.46(\mathrm{~m}, 2 \mathrm{H}), 0.16 \sim 0.15(\mathrm{~d}$, $J=4.0 \mathrm{~Hz}, 2 \mathrm{H}) ;{ }^{13} \mathrm{C} \mathrm{NMR}\left(100 \mathrm{MHz}, \mathrm{CDCl}_{3}\right) \delta: 163.9$, $152.6,144.3,135.4,132.4,132.2,130.8,130.4,124.0$, 45.0, 10.3, 3.5. HRMS (ESI) calcd for $\mathrm{C}_{13} \mathrm{H}_{14} \mathrm{ClN}_{4} \mathrm{O}[\mathrm{M}+$ $\mathrm{H}]^{+}$277.0856, found 277.0852 .

2-氯- $N$-(2-氯苄基)-6-(1H-1,2,4-三唑-1-基)苯甲酰胺 (7d): 收率 64\%. 浅黄色固体, m.p. $155 \sim 157{ }^{\circ} \mathrm{C} ;{ }^{1} \mathrm{H}$
NMR (400 MHz, $\left.\mathrm{CDCl}_{3}\right) \delta: 8.33(\mathrm{~s}, 1 \mathrm{H}), 7.83(\mathrm{~s}, 1 \mathrm{H})$, $7.49 \sim 7.28(\mathrm{~m}, 4 \mathrm{H}), 7.25 \sim 7.17(\mathrm{~m}, \mathrm{H}), 6.97 \sim 6.94(\mathrm{t}, J=$ $5.6 \mathrm{~Hz}, 1 \mathrm{H}), 4.54 \sim 4.53(\mathrm{~d}, J=6.0 \mathrm{~Hz}, 2 \mathrm{H}) ;{ }^{13} \mathrm{C} \mathrm{NMR}$ $\left(100 \mathrm{MHz}, \mathrm{CDCl}_{3}\right) \delta: 164.1,152.3,144.0,135.2,134.3$, $133.5,132.5,131.9,130.8,130.3,129.5,129.2,127.1$, 123.7, 41.86. HRMS (ESI) calcd for $\mathrm{C}_{16} \mathrm{H}_{13} \mathrm{Cl}_{2} \mathrm{~N}_{4} \mathrm{O}[\mathrm{M}+$ $\mathrm{H}]^{+}$347.0466, found 347.0462 .

2-氯- $N$-(4-氯苄基)-6-(1H-1,2,4-三唑-1-基)苯甲酰胺 (7e): 收率 58\%. 灰白色固体, m.p. $143 \sim 145{ }^{\circ} \mathrm{C} ;{ }^{1} \mathrm{H}$ NMR (400 MHz, $\left.\mathrm{CDCl}_{3}\right) \delta: 8.35(\mathrm{~s}, 1 \mathrm{H}), 7.85(\mathrm{~s}, 1 \mathrm{H})$, $7.48 \sim 7.39(\mathrm{~m}, 3 \mathrm{H}), 7.32 \sim 7.22(\mathrm{~m}, 4 \mathrm{H}), 6.83 \sim .80(\mathrm{t}, J=$ $5.8 \mathrm{~Hz}, 1 \mathrm{H}), 4.56 \sim 4.54(\mathrm{~d}, J=6.0 \mathrm{~Hz}, 2 \mathrm{H}) ;{ }^{13} \mathrm{C} \mathrm{NMR}$ $\left(100 \mathrm{MHz}, \mathrm{CDCl}_{3}\right) \delta: 164.0,152.4,144.2,135.5,135.2$, $133.5,132.4,132.0,130.9,130.4,129.2,128.8,123.8$, 43.2. HRMS (ESI) calcd for $\mathrm{C}_{16} \mathrm{H}_{12} \mathrm{Cl}_{2} \mathrm{~N}_{4} \mathrm{O}[\mathrm{M}+\mathrm{H}]^{+}$ 347.0466, found 347.0458.

2-氯- $N$-(4-甲氧基苄基)-6-(1H-1,2,4-三唑-1-基)苯甲 酰胺(7f): 收率 $68 \%$. 浅黄色固体, m.p. 152 $154{ }^{\circ} \mathrm{C} ;{ }^{1} \mathrm{H}$ NMR $\left(400 \mathrm{MHz}, \mathrm{CDCl}_{3}\right) \delta: 8.51(\mathrm{~s}, 1 \mathrm{H}), 8.01(\mathrm{~s}, 1 \mathrm{H})$, $7.53 \sim 7.44(\mathrm{~m}, 3 \mathrm{H}), 7.10 \sim 7.08(\mathrm{~d}, J=8.4 \mathrm{~Hz}, 2 \mathrm{H})$, $6.84 \sim 6.81(\mathrm{~d}, J=8.8 \mathrm{~Hz}, 2 \mathrm{H}), 6.15$ (brs, $1 \mathrm{H}), 4.43 \sim 4.42$ (d, $J=5.6 \mathrm{~Hz}, 2 \mathrm{H}), 3.79(\mathrm{~s}, 3 \mathrm{H}) ;{ }^{13} \mathrm{C} \mathrm{NMR}(100 \mathrm{MHz}$, $\left.\mathrm{CDCl}_{3}\right) \delta: 163.8,159.2,152.6,144.2,135.4,132.5,132.1$, 130.9, 130.4, 129.4, 128.8, 123.9, 114.1, 55.3, 43.6. HRMS (ESI) calcd for $\mathrm{C}_{17} \mathrm{H}_{16} \mathrm{ClN}_{4} \mathrm{O}_{2}[\mathrm{M}+\mathrm{H}]^{+}$343.0962, found 343.0954.

2-氯- $N, N$-二乙基-6-(1H-1,2,4-三唑-1-基)苯甲酰胺 (7g): 收率 56\%. 黄色固体, m.p. $135 \sim 137{ }^{\circ} \mathrm{C} ;{ }^{1} \mathrm{H}$ NMR $\left(400 \mathrm{MHz}, \mathrm{CDCl}_{3}\right) \delta: 8.60(\mathrm{~s}, 1 \mathrm{H}), 8.07(\mathrm{~s}, 1 \mathrm{H}), 7.54 \sim$ $7.47(\mathrm{~m}, 3 \mathrm{H}), 3.50 \sim 3.43(\mathrm{~m}, 2 \mathrm{H}), 3.09 \sim 2.93(\mathrm{~m}, 2 \mathrm{H})$, $1.13 \sim 1.10(\mathrm{t}, J=6.8 \mathrm{~Hz}, 3 \mathrm{H}), 0.89 \sim 0.85(\mathrm{t}, J=7.2 \mathrm{~Hz}$, $3 \mathrm{H}) ;{ }^{13} \mathrm{C} \mathrm{NMR}\left(100 \mathrm{MHz}, \mathrm{CDCl}_{3}\right) \delta: 164.3,152.5,144.3$, $134.8,131.8,131.5,130.3,130.1,123.8,42.9,39.1,13.3$, 12.2. HRMS (ESI) calcd for $\mathrm{C}_{13} \mathrm{H}_{16} \mathrm{ClN}_{4} \mathrm{O}[\mathrm{M}+\mathrm{H}]^{+}$ 279.1013 , found 279.1009 .

2-氯- $N$-乙基-6-(1H-1,2,4-三唑-1-基)苯甲酰胺(7h): 收率 45\%. 黄色固体, m.p. 128 $130{ }^{\circ} \mathrm{C} ;{ }^{1} \mathrm{H}$ NMR (400 $\left.\mathrm{MHz} \mathrm{CDCl}_{3}\right) \delta: 8.52(\mathrm{~s}, 1 \mathrm{H}), 8.08(\mathrm{~s}, 1 \mathrm{H}), 7.54 \sim 7.48(\mathrm{~m}$, $3 \mathrm{H}), 5.89$ (brs, $1 \mathrm{H}), 3.37 \sim 3.34(\mathrm{~m}, 2 \mathrm{H}), 1.10 \sim 1.06(\mathrm{t}$, $J=7.2 \mathrm{~Hz}, 3 \mathrm{H}) ;{ }^{13} \mathrm{C} \mathrm{NMR}\left(100 \mathrm{MHz}, \mathrm{CDCl}_{3}\right) \delta: 163.9$, $152.6,144.3,135.3,132.4,132.3,130.8,130.4,123.9$, 35.0, 14.4. HRMS (ESI) calcd for $\mathrm{C}_{11} \mathrm{H}_{12} \mathrm{ClN}_{4} \mathrm{O}[\mathrm{M}+\mathrm{H}]^{+}$ 251.0700 , found 251.0692

2-氯- $N$-苯基-6-(1H-1,2,4-三唑-1-基)苯甲酰胺(7i): 收率 $63 \%$. 黄色固体, m.p. 140 $143{ }^{\circ} \mathrm{C} ;{ }^{1} \mathrm{H}$ NMR (400 
$\left.\mathrm{MHz}, \mathrm{CDCl}_{3}\right) \delta: 8.53(\mathrm{~s}, 1 \mathrm{H}), 8.04(\mathrm{~s}, 1 \mathrm{H}), 7.80(\mathrm{~s}, 1 \mathrm{H})$, $7.56 \sim 7.45(\mathrm{~m}, 5 \mathrm{H}), 7.36 \sim 7.32(\mathrm{~m}, 2 \mathrm{H}), 7.19 \sim 7.16(\mathrm{dd}$, $J=7.2,7.6 \mathrm{~Hz}, 1 \mathrm{H}) ;{ }^{13} \mathrm{C}$ NMR $\left(100 \mathrm{MHz}, \mathrm{CDCl}_{3}\right) \delta$ : $162.1,152.7,144.2,136.7,135.4,132.7,132.1,131.2$, 130.6, 129.2, 125.5, 123.8, 120.6. HRMS (ESI) calcd for $\mathrm{C}_{15} \mathrm{H}_{12} \mathrm{ClN}_{4} \mathrm{O}[\mathrm{M}+\mathrm{H}]^{+}$299.0700, found 299.0694.

2-氯- $N$-(2-氯苯基)-6-(1H-1,2,4-三唑-1-基)苯甲酰胺 (7j): 收率 $73 \%$. 淡黄色固体, m.p. $125 \sim 127{ }^{\circ} \mathrm{C} ;{ }^{1} \mathrm{H}$ NMR (400 MHz, DMSO- $\left.d_{6}\right) \delta: 10.38(\mathrm{~s}, 1 \mathrm{H}), 8.91(\mathrm{~s}, 1 \mathrm{H})$, $8.25(\mathrm{~s}, 1 \mathrm{H}), 7.76 \sim 7.67(\mathrm{~m}, 3 \mathrm{H}), 7.56 \sim 7.48(\mathrm{~m}, 2 \mathrm{H})$, $7.38 \sim 7.34(\mathrm{~m}, 1 \mathrm{H}), 7.27 \sim 7.23(\mathrm{~m}, 1 \mathrm{H}) ;{ }^{13} \mathrm{C}$ NMR $(100$ MHz, DMSO- $\left.d_{6}\right) \delta: 162.8,152.8,145.2,135.7,134.4$, $132.3,132.0,131.7,130.3,130.1,128.0,127.9,127.7$, 127.5, 123.9. HRMS (ESI) calcd for $\mathrm{C}_{15} \mathrm{H}_{11} \mathrm{Cl}_{2} \mathrm{~N}_{4} \mathrm{O}[\mathrm{M}+$ $\mathrm{H}]^{+}$333.0310, found 333.0302 .

2-氯- $N$-(2-氟苯基)-6-( $1 H-1,2,4$-三唑-1-基)苯甲酰胺 (7k): 收率 66\%. 淡黄色固体, m.p. $169 \sim 171{ }^{\circ} \mathrm{C} ;{ }^{1} \mathrm{H}$ NMR (400 MHz, DMSO- $\left.d_{6}\right) \delta: 10.51(\mathrm{~s}, 1 \mathrm{H}), 8.90(\mathrm{~s}, 1 \mathrm{H})$, $8.21(\mathrm{~s}, 1 \mathrm{H}), 7.73 \sim 7.69(\mathrm{~m}, 4 \mathrm{H}), 7.26 \sim 7.19(\mathrm{~m}, 3 \mathrm{H}) ;{ }^{13} \mathrm{C}$ NMR (100 MHz, DMSO- $\left.d_{6}\right) \delta: 162.7,154.7$ (d, $J=245.5$ Hz), 152.8, 145.1, 135.7, 132.3, 132.0, 131.7, 130.3, 126.9 (d, $J=7.4 \mathrm{~Hz}), 125.5$ (d, $J=5.9 \mathrm{~Hz}), 125.4(\mathrm{~d}, J=6.2 \mathrm{~Hz})$, $124.8(\mathrm{~d}, J=3.6 \mathrm{~Hz}), 123.9,116.2(\mathrm{~d}, J=19.0 \mathrm{~Hz})$. HRMS (ESI) calcd for $\mathrm{C}_{15} \mathrm{H}_{11} \mathrm{ClFN}_{4} \mathrm{O}[\mathrm{M}+\mathrm{H}]^{+} 317.0605$, found 317.0603 .

2-氯- $N$-(2-氧苯基)-6-(1H-1,2,4-三唑-1-基)苯甲酰胺 (71): 收率 60\%. 亚白色固体, 熔点 $179 \sim 181{ }^{\circ} \mathrm{C} ;{ }^{1} \mathrm{H}$ NMR (400 MHz, DMSO- $\left.d_{6}\right) \delta: 10.93(\mathrm{~s}, 1 \mathrm{H}), 8.97(\mathrm{~s}, 1 \mathrm{H})$, $8.22(\mathrm{~s}, 1 \mathrm{H}), 7.84 \sim 7.71(\mathrm{~m}, 5 \mathrm{H}), 7.57 \sim 7.55$ (d, $J=7.6$ $\mathrm{Hz}, 1 \mathrm{H}), 7.42 \sim 7.38(\mathrm{~m}, 1 \mathrm{H}) ;{ }^{13} \mathrm{C}$ NMR $(100 \mathrm{MHz}$, DMSO- $\left.d_{6}\right) \delta: 163.0,152.9,145.1,139.7,135.7,134.3$, $134.0,132.2,131.9,131.6,130.3,126.8,126.1,123.5$, 116.9, 107.6. HRMS (ESI) calcd for $\mathrm{C}_{16} \mathrm{H}_{11} \mathrm{ClN}_{5} \mathrm{O}[\mathrm{M}+$ $\mathrm{H}]^{+}$324.0652, found 324.0644 .

3.3.5 2-氯- $N$-(2-氯芳基)-6-(5-硫代-2,5-二氢- $1 H$ 1,2,4-三唑-1-基)苯甲酰胺 (8) 的合成

取 $0.24 \mathrm{~g}(0.7 \mathrm{mmol}) 2$-氯- $N$-(2-氯苄基)-6-(1H-1,2,4三唑-1-基)苯甲酰胺(7d)加入到三烧瓶中, 加入 $10 \mathrm{~mL}$ $\mathrm{N}, \mathrm{N}$-二甲基甲酰胺(DMF)到反应瓶中, 然后加入 $0.23 \mathrm{~g}$ $\left(7 \mathrm{mmol}\right.$ )硫粉在 $160{ }^{\circ} \mathrm{C}$ 回流反应 $12 \mathrm{~h}$, 在反应过程中, 随着温度升高, 硫粉逐渐溶解, 溶液颜色加深. 通过 TLC 检测直至反应完全. 冷却负压过滤未反应的硫粉, 然后用水洗, 乙酸乙酯萃取, 有机相用无水硫酸钠干燥 后浓缩, 残余物用薄层制备色谱[氯仿/甲醇, $V: V=$ $90: 1$ ]分离得到 $80.11 \mathrm{~g}$, 收率 41\%. 浅黄色固体, m.p.
76 $77{ }^{\circ} \mathrm{C} ;{ }^{1} \mathrm{H}$ NMR $\left(400 \mathrm{MHz}, \mathrm{CDCl}_{3}\right) \delta: 7.68(\mathrm{~s}, 1 \mathrm{H})$, $7.57 \sim 7.56(\mathrm{~m}, 1 \mathrm{H}), 7.49 \sim 7.48(\mathrm{~m}, 3 \mathrm{H}), 7.33 \sim 7.31(\mathrm{~m}$, 1H), $7.20 \sim 7.18(\mathrm{~m}, 2 \mathrm{H}), 7.05$ (brs, $1 \mathrm{H}), 4.66 \sim 4.65$ (d, $J=6.0 \mathrm{~Hz}, 2 \mathrm{H}) ;{ }^{13} \mathrm{C}$ NMR $\left(100 \mathrm{MHz}, \mathrm{CDCl}_{3}\right) \delta: 167.1$, $164.0,139.2,135.4,134.7,134.4,133.7,132.5,131.5$, $130.8,130.7,129.4,129.2,127.2,127.1,41.9$. HRMS (ESI) calcd for $\mathrm{C}_{16} \mathrm{H}_{13} \mathrm{Cl}_{2} \mathrm{~N}_{4} \mathrm{OS}[\mathrm{M}+\mathrm{H}]^{+}$379.0187, found 379.0181 .

\section{4 三唑苯甲酰胺化合物的生物活性测试}

合成的 1,2,4-三唑苯甲酰胺衍生物的生物活性采用 菌丝生长速率法测试 ${ }^{[29]}$. 首先, 称取 $200 \mathrm{~g}$ 去皮后的新 鲜土豆, 用 $1 \mathrm{~L}$ 蒸馏水加热煮沸 $20 \mathrm{~min}$, 用纱布滤出未 煮烂的土豆，向滤液中加入葡萄糖 $20 \mathrm{~g}$ 和琼脂 $15 \mathrm{~g}$, 充 分搅拌待其完全溶解后，用蒸馏水定容到 $1 \mathrm{~L}$. 接着在 $120{ }^{\circ} \mathrm{C}$ 下湿热灭菌 $20 \mathrm{~min}$, 冷却制得马铃薯葡萄糖琼脂 (PDA)培养基.

然后，以二甲亚砜(DMSO)为溶剂配制适当浓度的 供试样品, 并加入适当数量的表面活性剂吐温备用. 移 取适当浓度的 $1 \mathrm{~mL}$ 供试样品溶液, 和熔化并冷却至 $50{ }^{\circ} \mathrm{C}$ 的 $9 \mathrm{~mL}$ PDA 培养基充分混合, 然后倒入无菌培 养皿中冷却至室温. 把在 PDA 平板上培养 $7 \mathrm{~d}$ 的小麦全 蚀病病原菌用直径 $5 \mathrm{~mm}$ 的打孔器制作成菌饼, 然后将 菌饼以倒扣的方式接种在含药的 PDA 平板中央, 以仅 含 DMSO 的平板培养基为对照. 每个药液浓度设 4 个重 复, 在 $20 \sim 25{ }^{\circ} \mathrm{C}$ 培养箱中避光培养 $5 \mathrm{~d}$, 调查菌落直径, 计算相对抑制率 $[$ 相对抑制率 $(\%)=($ 空白对照菌落直径药剂处理菌落直径)/空白对照菌落直径 $\times 100 \%$ ]. 假禾 谷镰孢菌活性测试采用相似的方法.

辅助材料(Supporting Information) 所涉及目标化合 物及中间体的 ${ }^{1} \mathrm{H} \mathrm{NMR}$ 和 ${ }^{13} \mathrm{C}$ NMR 数据. 这些材料可以 免费从本刊网站(http://sioc-journal.cn/)上下载.

\section{References}

[1] Xu, M. S.; Guo, S.; Yang, F. P.; Wang, Y.; Wu, C. H.; Jiang, X. R.; Zhao, Q. J.; Chen, W. M.; Tian, G. H.; Zhu, F. Q.; Xie, Y. C.; Hu, T. W.; Wang, Z.; He, Y.; Shen, J. S. Arch. Pharm. Chem. Life Sci. 2019, 352, e1800306.

[2] Lu, A.; Luo, H.; Shi, M.; Wu, G.; Yuan, Y.; Liu, J.; Tang, F. Bioorg. Med. Chem. Lett. 2011, 21, 4924.

[3] Zang, J.; Liang, X. W.; Huang, Y. X.; Jia, Y. P.; Li, X. Y.; Xu, W. F.; Chou, C. J.; Zhang, Y. J. J. Med. Chem. 2018, 61, 5304.

[4] Huang, L.; Lai, W. H.; Zhu, L.; Li, W.; Wei, L.; Lee, K. H.; Xie, L.; Chen, C. H. ACS Med. Chem. Lett. 2018, 9, 268.

[5] Shi, J.-J.; Ren, G.-H.; Wu, N.-J.; Liu, X.-H.; Xu, T.-M.; Tan, C.-X. Chin. J. Org. Chem. 2017, 37, 2131 (in Chinese). (史建俊, 任贵华, 吴宁捷, 刘幸海, 许天明, 谭成侠, 有机化学, 2017, 37, 2131.)

[6] Zhang, J. F.; Xu, J. Y.; Wang, B. L.; Li, Y. X.; Xiong, L. X.; Li, Y. Q.; Ma, Y.; Li, Z. M. J. Agric. Food Chem. 2012, 60, 7565.

[7] Chen, K.; Liu, Q.; Ni, P. J.; Zhu, H. J.; Lia, Y. F.; Wang, Q. Pest 
Manage. Sci. 2015, 71, 1503.

[8] Wang, M.-M.; Zhang, Q.-Q.; Yue, K.; Li, Q.-S.; Xu, F.-B. Chin. J. Org. Chem. 2017, 37, 1774 (in Chinese)

(王梦梦, 张青青, 岳凯, 李庆山, 徐风波, 有机化学, 2017, 37, 1774.)

[9] Lahm, G. P.; Stevenson, T. M.; Selby, T. P.; Freudenberger, J. H.; Cordova, D.; Flexner, L.; Bellin, C. A.; Dubas, C. M.; Smith, B. K.; Hughes, K. A.; Hollingshaus, J. G.; Clark, C. E.; Benner, E. A. Bioorg. Med. Chem. Lett. 2007, 17, 6274.

[10] Hughes, K. A.; Lahm, G. P.; Selby, T. P.; Stevenson, T. M. WO 2004067528, 2004 [Chem. Abstr. 2004, 141, 190786].

[11] Li, W.-M; Wang, J.-G.; Li, Y.-H.; Wang, S.-H.; Li, Z.-M. Chem. J. Chin. Univ. 2010, 31, 1574 (in Chinese). (李文明, 王建国, 李永红, 王素华, 李正名, 高等学校化学学 报, 2010, 31, 1574.)

[12] Yang, J.-C.; Zhang, J.-B.; Chai, B.-S.; Liu, C.-L. Agrochemicals 2008, 47, 6 (in Chinese) (杨吉春, 张金波, 柴宝山, 刘长令, 农药, 2008, 47, 6.)

[13] Eicken, K.; Goetz, N.; Harreus, A.; Ammermann, E.; Lorenz, G.; Rang, H. EP 545099, 1993 [Chem. Abstr. 1993, 119, 160132].

[14] Moradi, W. A.; Schlegel, G.; Schnatterer, A.; Volz, F. WO 2016173998, 2016 [Chem. Abstr. 2016, 165, 571164].

[15] Yoshikawa, Y.; Tomitani, K.; Katsuta, H.; Kawashima, H.; Takahashi, T.; Inami, S.; Yanase, Y.; Takashi, A.; Shimotori, H.; Tomura, N. JP 09301974, 1997 [Chem. Abstr. 1997, 128, 22908].

[16] Phillion, D.; Wong, S. C.; Shortt, B. US 5486621, 1996 [Chem. Abstr. 1996, 124, 253325].

[17] Freeman, J.; Ward, E. Mol. Plant Pathol. 2004, 5, 235.

[18] Xie, G.-Y.; Jin, W.-B.; Zhao, Y.-Q.; Cheng, Y.-N.; Sun, B.-J.; Sun, S.-J.; Wang, M.-Z.; Wei, D.-D.; Li, H.-L. Chin. J. Org. Chem. 2014,
34, 1124 (in Chinese).

（谢桂英，靳文波，赵艳芹，程绎南，孙炳剑，孙淑君，汪梅子， 位丹丹，李洪连，有机化学, 2014, 34, 1124.)

[19] Jin, W.-B.; Xie, G.-Y.; Sun, S.-J.; Zhao, Y.-Q.; Cheng, Y.-N.; Sun, B.-J.; Li, H.-L. Chin. J. Org. Chem. 2014, 34, 2376 (in Chinese). (靳文波, 谢桂英, 孙淑君, 赵艳芹, 程绎南, 孙炳剑, 李洪连, 有机化学, 2014, 34, 2376.)

[20] Banerjee, S.; Ganguly, S.; Sen, K. K. J. Adv. Pharm. Educ. Res. 2013, 3, 102 .

[21] Antilla, J. C.; Baskin, J. M.; Barder, T. E.; Buchwald, S. L. J. Org. Chem. 2004, 69, 5578.

[22] Hosseini-Sarvari, M.; Razmi, Z. RSC Adv. 2014, 4, 44105.

[23] Hu, S.-S.; Guo, H.-C.; Jiang, H.-J.; Zheng, R.-H. Chin. J. Appl. Chem. 2011, 28, 1179 (in Chinese).

(胡帅帅，郭海昌，蒋华江，郑人华，应用化学, 2011, 28, 1179.)

[24] Yang, K.; Qiu, Y. T.; Li, Z.; Wang, Z. Y.; Jiang, S. J. Org. Chem. 2011, 76, 3151.

[25] Lee, S. H.; Kim, M. J.; Lee, S. H.; Kim, J.; Park, H. J.; Lee, J. Eur. J. Med. Chem. 2011, 46, 2662.

[26] Miyamoto, H.; Ueda, H.; Otsuka, T.; Aki, S.; Tamaoka, H.; Tominaga, M.; Nakagawa, K. Chem. Pharm. Bull. 1990, 38, 2472.

[27] Tsukamoto, I.; Koshio, H.; Akamatsu, S.; Kuramochi, T.; Saitoh, C.; Yatsu, T.; Yanai-Inamura, H.; Kitada, C.; Yamamoto, E.; Sakamoto, S.; Tsukamoto, S. Bioorg. Med. Chem. 2008, 16, 9524

[28] Jautelat, M.; Erdman, D. DE 19744706, 1999 [Chem. Abstr. 1999, $130,267441]$.

[29] Wang, L.; Zhang, Y.; Wang, D.; Wang, M.; Wang, Y.; Feng, J. J. Agric. Food Chem. 2018, 66, 81.

[30] Jautelat, M.; Tiemann, R.; Dutzmann, S.; Haensler, G.; Stenzel, K. DE 19528046, 1996 [Chem. Abstr. 1996, 125, 114638].

(Lu, Y.) 